\title{
GRB 090926A AND BRIGHT LATE-TIME FERMI LARGE AREA TELESCOPE GAMMA-RAY BURST AFTERGLOWS
}

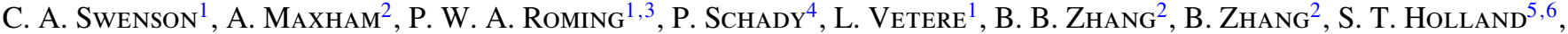 \\ J. A. Kennea ${ }^{1}$, N. P. M. Kuin ${ }^{7}$, S. R. OAtes ${ }^{7}$, K. L. Page ${ }^{8}$, And M. De Pasquale ${ }^{7}$ \\ ${ }^{1}$ Pennsylvania State University, 525 Davey Lab, University Park, PA 16802, USA; cswenson@ astro.psu.edu \\ ${ }^{2}$ University of Nevada, Las Vegas, Box 454002, 4505 Maryland Parkway, Las Vegas, NV 89154, USA \\ ${ }^{3}$ Southwest Research Institute, 6220 Culabra Road, San Antonio, TX 78238, USA \\ ${ }^{4}$ Max-Planck-Institut für extraterrestrische Physik, Garching, Germany \\ ${ }^{5}$ Universities Space Research Association, 10227 Wincopin Circle, Suite 500, Columbia, MD 21044, USA \\ ${ }^{6}$ Center for Research and Exploration in Space Science and Technology, Code 668.8, Greenbelt, MD 20771, USA \\ 7 The UCL Mullard Space Science Laboratory, Holmbury St Mary, Surrey, RH5 6NT, UK \\ ${ }^{8}$ Department of Physics and Astronomy, University of Leicester, University Road, Leicester LE1 7RH, UK \\ Received 2010 April 28; accepted 2010 June 11; published 2010 June 28
}

\begin{abstract}
GRB 090926A was detected by both the Gamma-ray Burst Monitor and Large Area Telescope (LAT) instruments on board the Fermi Gamma-ray Space Telescope. Swift follow-up observations began $\sim 13 \mathrm{hr}$ after the initial trigger. The optical afterglow was detected for nearly 23 days post trigger, placing it in the long-lived category. The afterglow is of particular interest due to its brightness at late times, as well as the presence of optical flares at $\mathrm{T} 0+10^{5} \mathrm{~s}$ and later, which may indicate late-time central engine activity. The LAT has detected a total of 16 gamma-ray bursts; nine of these bursts, including GRB 090926A, also have been observed by Swift. Of the nine Swift-observed LAT bursts, six were detected by UVOT, with five of the bursts having bright, long-lived optical afterglows. In comparison, Swift has been operating for five years and has detected nearly 500 bursts, but has only seen $\sim 30 \%$ of bursts with optical afterglows that live longer than $10^{5} \mathrm{~s}$. We have calculated the predicted gamma-ray fluence, as would have been seen by the Burst Alert Telescope (BAT) on board Swift, of the LAT bursts to determine whether this high percentage of long-lived optical afterglows is unique, when compared to BAT-triggered bursts. We find that, with the exception of the short burst GRB 090510A, the predicted BAT fluences indicate that the LAT bursts are more energetic than $88 \%$ of all Swift bursts and also have brighter than average X-ray and optical afterglows.
\end{abstract}

Key words: gamma-ray burst: general - gamma-ray burst: individual (GRB 090926A) - shock waves

Online-only material: color figures

\section{INTRODUCTION}

The Fermi Gamma-ray Space Telescope has opened a new era of gamma-ray burst (GRB) observations. With the onboard Gamma-ray Burst Monitor (GBM) and Large Area Telescope (LAT) instruments (Atwood et al. 2009; Meegan et al. 2009), GRB prompt emission can be probed at higher energies than ever before. Used in conjunction with Swift (Gehrels et al. 2004), GRB afterglows can be studied across a nearly continuous band from $\mathrm{GeV}$ energies to optical wavelengths. As of 2010 April 1, LAT has detected 16 GRBs, one of which was simultaneously localized by the Swift Burst Alert Telescope (BAT; Barthelmy et al. 2005), and eight others had Swift follow-up observations at late times. The Swift X-ray Telescope (XRT; Burrows et al. 2005a) detected the afterglow from seven of the nine LAT bursts; six of which were detected by the Swift UV/Optical Telescope (UVOT; Roming et al. 2005). All but one of these UVOT afterglows stand out due to their brightness and length of detectability.

During the first five years of operation, Swift detected $\sim 500$ GRBs, but $\lesssim 30 \%$ had bright, long-lived optical afterglows that extended beyond $10^{5} \mathrm{~s}$. Comparing Swift and LAT GRBs, twothirds of LAT bursts with follow-up observations have optical afterglows that rival the brightest and longest lived of the Swift sample. Such a high percentage raises the question as to whether LAT bursts differ from the Swift sample. Two possibilities are that LAT is observing GRBs that exhibit extended energy injection, resulting in bright optical afterglows at late times, or
LAT bursts could simply be brighter, at all wavelengths, than the "average" BAT-triggered burst allowing for later detections of the afterglow (see Gehrels et al. 2008).

GRB 090926A is an LAT-detected burst with a bright, long-lived UVOT afterglow. In this Letter, we present the multiwavelength study of GRB 090926A, examining the X-ray and UV/optical wavelengths as observed by Swift. In an attempt to understand the high percentage of LAT-detected bursts with optical afterglows, we also use the Fermi observations of the prompt emission to calculate the expected fluence as would have been observed by the BAT. We perform this same calculation for the six other LAT bursts also detected by XRT and compare them to a sample of BAT-triggered bursts.

We use the power-law representation of flux density, $f_{v}(t) \propto$ $t^{\alpha} v^{\beta}$, where $\alpha$ and $\beta$ are the temporal and spectral indices, respectively. Errors are reported at $1 \sigma$, unless otherwise specified.

\section{OBSERVATIONS AND DATA REDUCTION}

\subsection{Fermi Data}

At 04:20:26.99 UT on 2009 September 26, GBM triggered on GRB 090926A (Bissaldi 2009). The GBM light curve, Figure 1, consisted of a single pulse with $T_{90}$ of $20 \pm 2 \mathrm{~s}(8-1000 \mathrm{keV})$. The time-averaged GBM/LAT spectrum from T0 to T0+20.7 s, where $\mathrm{T} 0$ is the trigger time, is best fit by a Band function (Band et al. 1993), with $E_{\mathrm{p}}=268 \pm 4 \mathrm{keV}, \alpha=-0.693 \pm 0.009$, and $\beta=-2.342 \pm 0.011$. The fluence $(10 \mathrm{keV}-10 \mathrm{GeV})$ during this interval is $(2.47 \pm 0.03) \times 10^{-4} \mathrm{erg} \mathrm{cm}^{-2}$, bright enough 


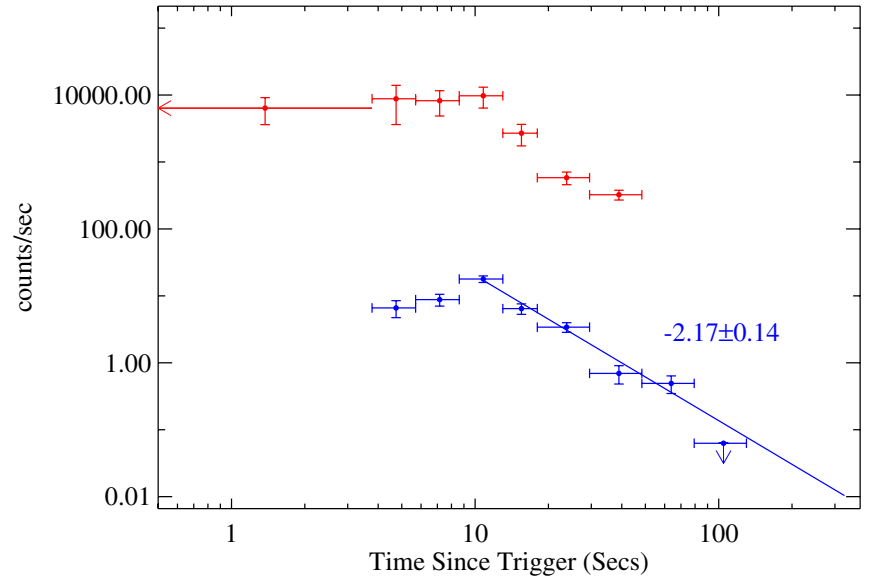

Figure 1. Fermi GBM (upper) and LAT (lower) light curves. (A color version of this figure is available in the online journal.)

to result in a Fermi repointing. In the first $300 \mathrm{~s}$, LAT observed 150 and 20 photons above $100 \mathrm{MeV}$ and $1 \mathrm{GeV}$, respectively. Possible extended emission continued out to a few kiloseconds. The highest energy photon, $19.6 \mathrm{GeV}$, was observed $26 \mathrm{~s}$ after the trigger. The LAT light curve, Figure 1, is fit by a power law of $\alpha=-2.17 \pm 0.14$. We fit the LAT spectrum, from 100-1000 s, with a power law of $\beta=-1.26_{-0.22}^{+0.24}$.

\subsection{XRT Data}

XRT began observing GRB 090926A 46.6 ks after the Fermi trigger. The light curve, Figure 2 (Evans et al. 2007, 2009), shows a decaying behavior with some evidence of variability and is fit with a single power law, decaying with $\alpha=-1.40 \pm 0.05$ ( $90 \%$ confidence level). The average spectrum from $46.6-149 \mathrm{ks}$ is best fit by an absorbed power-law model with $\beta=-1.6_{-0.2}^{+0.3}$ and an absorption column density of $1.0_{-0.3}^{+0.5} \times 10^{21} \mathrm{~cm}^{-2}$ in excess of the Galactic value of $2.7 \times 10^{20} \mathrm{~cm}^{-2}$ (Kalberla et al. 2005). The counts to observed flux conversion factor deduced from this spectrum is $3.5 \times 10^{-11} \mathrm{erg} \mathrm{cm}^{-2}$ count $^{-1}$. The average observed (unabsorbed) fluxes are 1.3(1.9) $\times 10^{-12} \mathrm{erg} \mathrm{cm}^{-2} \mathrm{~s}^{-1}$.

\subsection{UVOT Data}

UVOT began settled observations of GRB 090926A at T0+ $47 \mathrm{ks}$, and the optical afterglow was immediately detected (Gronwall \& Vetere 2009). The resulting light curve is shown in Figure 2. After removing the flares, the underlying optical light curve is well fit $\left(\chi_{\text {red }}^{2}=0.92 / 82\right.$ degrees of freedom $)$ by a broken power law. The best fit parameters are $\alpha_{\mathrm{Opt}, 1}=-1.01_{-0.03}^{+0.07}$, $t_{\text {break }}=351_{-141.9}^{+70.2} \mathrm{ks}, \alpha_{\mathrm{Opt}, 2}=-1.77_{-0.26}^{+0.21}$. X-shooter, mounted on the Very Large Telescope UT2, found a spectroscopic redshift of $z=2.1062$ (Malesani et al. 2009).

\subsection{Flaring Activity}

Variability in the X-ray is not statistically strong (peaking $\sim 2.8 \sigma$ above the underlying fit) but is temporally coincident with stronger flaring in the UVOT. The first flare, at $\sim 70-95 \mathrm{ks}$, is well defined in the UVOT light curve with $\Delta t / t \approx 0.35$, but is only seen in the X-ray as minor variability, with individual points varying from the underlying fit. The second flare, at 195-260 ks, is better defined in the X-ray (though only peaking at $\sim 1 \sigma$ ) but is matched by a similarly shaped, stronger feature in the $\operatorname{UVOT}(\Delta t / t \approx 0.28)$. Due to an observing gap, we may not have

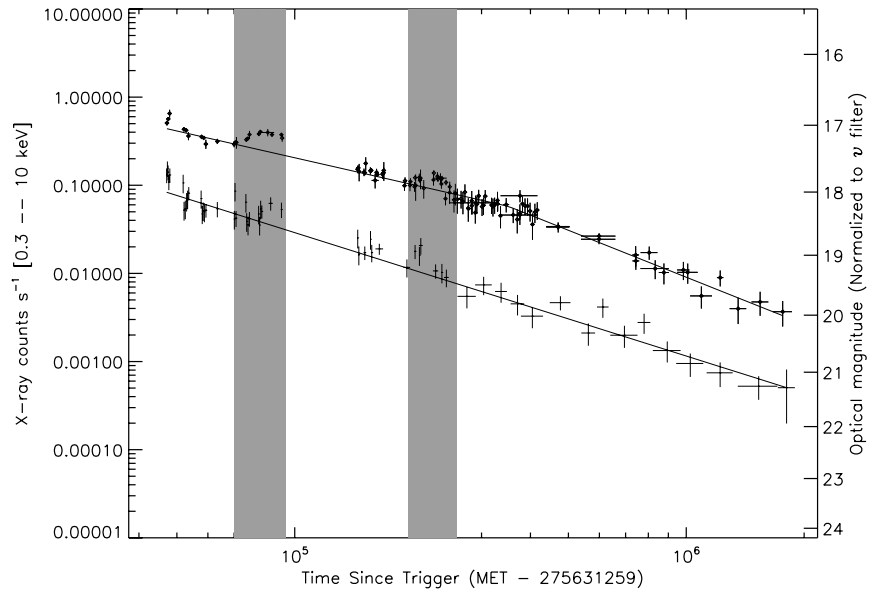

Figure 2. Light curves for the XRT (bottom) and UVOT (top). Shaded regions indicate periods of flaring.

observed the peak of the UVOT feature, but it appears to lag the peak of the X-ray feature by $\sim 6 \mathrm{ks}$, which is consistent with lower energy emission from flares lagging the higher energy (Margutti et al. 2010).

\section{DISCUSSION}

GRB 090926A was a long burst with more than 20 photons in the $\mathrm{GeV}$ range, which was easily detected by XRT and UVOT $\sim 13 \mathrm{hr}$ after the trigger and has late-time flares in the UVOT afterglow. The overall brightness and behavior of the optical afterglow are more reminiscent of afterglows observed immediately after the trigger, as opposed to observations starting $47 \mathrm{ks}$ after the trigger (Oates et al. 2009; Roming et al. 2009; P. W. A. Roming et al. 2010, in preparation). The late-time light curve could be due to late-time energy injection, supported by the presence of flares in the light curve, or could be an LAT selection effect. We explore both of these possibilities.

\subsection{GRB 090926A Late-time Flares}

$\mathrm{X}$-ray flares at late times have been attributed to two different sources (Wu et al. 2005): central engine powered internal emission, or features of the external shock. There is evidence suggesting that the GRB prompt emission and X-ray flares originate from similar physical processes (see Burrows et al. 2005b; Zhang et al. 2006; Chincarini et al. 2007; Krimm et al. 2007), including a lower energy budget and "spiky" flares more like those actually seen in X-ray light curves. In the case of LATdetected GRBs, flares requiring large energies would result in an unrealistic combined energy budget (prompt + flares). A low-energy mechanism that does not significantly affect the prompt energy is required. This makes the central engine a likely candidate for the observed flares.

If the central engine is the source of GRB flares, the X-ray flare spectrum should be similar to that of the prompt spectrum. In the case of GRB 090926A, the prompt emission was seen to have a Band-function spectrum. Assuming the optical behaves similarly to the X-ray and that the flares are caused by central engine activity, we would expect a Band-function spectrum during the flares. A Band-function spectrum is not observed during the X-ray variability or optical flares. The flares are both well fit by a power law, with no indication of a break in the spectrum or sign of spectral evolution in the X-ray. However, the statistics of the X-ray light curve are low enough that detecting a 
Band spectrum may not be possible, even if it exists. Combining the poor statistics with the dominant underlying continuum, it is not surprising that a power law is the best fit. We also find no evidence of change in the spectral shape after creating a spectral energy distribution using optical/UV photometry before and during the first flare.

A non-Band-like spectrum for the flares does not expressly prohibit central engine activity from being the source of the flares. Code for modeling X-ray flares in GRBs (Maxham \& Zhang 2009) can produce optical flares through the collision of low-energy shells or wide shells. If the two flares are indeed due to internal shocks, then this code can put constraints on the time of ejection and maximum energy (Lorentz factor) of the matter shells that could produce such flares. Since ejection time in the GRB rest frame is highly correlated to the collision time of shells in the observer frame, this means that the central engine is active around 70 and $197 \mathrm{ks}$. Using the prompt emission fluence to constrain the total energy contained in the blastwave, the internal shock model requires that Lorentz factors of the shells causing flares must be less than the Lorentz factor of the blastwave when the shells are ejected. Fast moving shells will simply collide into the blastwave giving small, undetectable glitches, whereas slow moving shells will be allowed to collide internally, releasing the energy required to detect a flare. Specifically, we find maximum Lorentz factors of $8.2\left(\frac{E_{52.3}}{n}\right)^{1 / 8}$ and $5.5\left(\frac{E_{52.3}}{n}\right)^{1 / 8}$ for the first and second flares, respectively, and in terms of the energy in the prompt emission in units of $10^{52.3} \mathrm{erg}$ and number density of the ambient medium.

Collisions between these relatively low-energy shells are expected to be seen in the lower energy UV/optical bands. In the synchrotron emission model, $E_{p}=2 \Gamma \gamma_{e} 2 \frac{\hbar e B}{m_{e} c} \propto L^{1 / 2}$ for electrons moving with a bulk Lorentz factor $\Gamma$ with typical energy $\gamma_{e} m_{e} c^{2}$, since the comoving magnetic field $B \propto L^{1 / 2}$ (Zhang \& Mészáros 2002). This is consistent with the empirical Yonetoku relation $E_{p} \propto L_{\text {iso }}^{1 / 2}$ (Yonetoku et al. 2004) for prompt GRB emission. Applying this relation to the two flares, one predicts $E_{p}$ of 0.8 and $0.5 \mathrm{eV}$ for each flare, respectively. This is consistent with the observation that both flares are more prominent in the optical band than in the X-ray band. Finding $E_{p}$ using the Amati relation, $E_{p} \propto E_{\text {iso }}^{1 / 2}$, (Amati et al. 2002) gives $E_{p}$ values for both flares around $1 \mathrm{keV}$, which are inconsistent with the observation. Unlike for individual burst pulses (whose durations do not vary significantly), which seem to follow an Amati relation (Krimm et al. 2009), the Yonetoku relation may be more relevant for flares because it is consistent with the more generic synchrotron emission physics. Since the duration of a flare depends on the epoch of the flare, the Amati relation is not expected to hold.

\subsection{Are LAT Bursts Brighter than Average?}

Despite its remarkably bright, late detection, GRB 090926 is not the first optical counterpart to be found at such late times. Since the launch of Fermi, Swift has performed follow-up observations of LAT-observed bursts: GRBs 080916C, 081024B, 090217, 090323, 090328, 090902B, 090926A, and 091003; all but GRB 081024 were long GRBs. None of these bursts were observed before $\sim 39 \mathrm{ks}$. Although Swift observations were performed as soon as possible, the error circle of the GBM is too large to be effectively observed by $S$ wift, therefore the more precise LAT position was required before observations could take place. Despite these delays, an X-ray counterpart was discovered by XRT for six of the eight bursts with follow-up observations.
UVOT detected an optical afterglow associated with five of the X-ray counterparts. In addition, the short GRB 090510A was a coincident trigger between GBM/LAT and BAT, raising the total number of Swift-observed LAT bursts to nine. GRB 090510A had both an X-ray and a UV/optical counterpart.

The high percentage of LAT-detected bursts with optical afterglows, when compared to the sample of Swift-triggered bursts, raises questions about the nature of the bursts themselves. Is the LAT preferentially sensitive to bursts that are brighter overall, resulting in a higher probability of detecting a bright, long-lived optical counterpart, or are the bursts themselves different, with a late-time brightening causing the optical afterglows?

The emission of $\mathrm{GeV}$ photons can come from several prompt and afterglow models (see Zhang 2007), but at low photon numbers. The fact that LAT has detected GeV photons suggests that the overall prompt flux must be very high. In conjunction with this, higher GeV flux would be associated with GRBs having a higher $E_{p}$, which have been shown to be correlated with higher luminosity GRBs (Amati et al. 2002; Yonetoku et al. 2004). These arguments would lead us to believe that LAT GRBs may be among the brightest GRBs ever observed (Rau et al. 2010).

To further investigate this, we calculated the fluence that would have been observed by the BAT for the bursts that were detected by LAT and later detected by XRT. Because we are assuming, for the purpose of this test, that the spectrum is brighter at all wavelengths, a bright LAT burst corresponds to a bright GBM burst. Under this assumption, we use the GBM spectral parameters provided by Ghisellini et al. (2010) to predict what would have been seen by the BAT over the $15-150 \mathrm{keV}$ range. We check our results and estimate our error by comparing the predicted and observed fluence for the simultaneously observed Fermi/Swift GRB 090510A. The GBM spectral parameters as well as the predicted BAT fluence between $15-150 \mathrm{keV}$ are shown in Table 1.

We limit our error in the calculation of the expected BAT fluence to the error introduced from the GBM parameters. Comparing the $T_{90}$ of GRB 090510A as observed by GBM and BAT ( $1 \mathrm{~s}$ and $0.3 \mathrm{~s}$, respectively), we realize that a certain amount of error will be introduced into the expected BAT fluence due to differences that would exist in the observed $T_{90}$ between the two instruments. In the case of the long bursts, this error is negligible in comparison to the GBM parameter errors. Because GRB 090510A is a short burst, a small difference in $T_{90}$ results in a proportionally larger error than a difference in a few seconds for longer bursts. However, our calculated value of the fluence for GRB 090510A differs by less than a factor of 2 from the BAT-observed value.

We compare the calculated fluences to a sample of 343 BATtriggered bursts from 2005 April to 2009 June. The sample is comprised of both short and long bursts, across a wide range of energies. The percentile ranking as a function of fluence is shown in Figure 3. All but one of the LAT-detected bursts are brighter than $88 \%$ of the BAT sample of bursts. The exception is the short burst, GRB 090510A.

Ukwatta et al. (2009) reported possible soft, extended emission associated with GRB 090510A. Because it was at a higher redshift than most short GRBs, $z=0.903$ (McBreen et al. 2010), BAT could not confirm any extended emission (De Pasquale et al. 2010). When we compare GRB 090510A to the BATtriggered extended emission short GRBs, we find that it is only brighter than $18 \%$ of the sample. If extended emission is in 
Table 1

The Seven LAT-observed Bursts That Have Been Observed by Swift and Detected by the XRT

\begin{tabular}{lcccccc}
\hline \hline \multicolumn{1}{c}{ GRB } & $\begin{array}{c}S_{\mathrm{GBM}} \\
\left(8-10^{4} \mathrm{keV}\right)\end{array}$ & $\begin{array}{c}T_{90} \\
(\mathrm{~s})\end{array}$ & $\beta_{1 \mathrm{GBM}}$ & $\beta_{2 \mathrm{GBM}}$ & $\begin{array}{c}E_{\mathrm{p}} \\
(\mathrm{keV})\end{array}$ & $\begin{array}{c}S_{\mathrm{BAT}} \\
(15-150 \mathrm{keV})\end{array}$ \\
\hline $080916 \mathrm{C}$ & $(1.6 \pm 0.2) \times 10^{-4}$ & 66 & $-0.91 \pm 0.02$ & $-2.08 \pm 0.06$ & $424 \pm 24$ & $1.735 \times 10^{-5}$ \\
090323 & $(1.32 \pm 0.03) \times 10^{-4}$ & $\sim 150$ & $-0.89 \pm 0.03$ & $\cdots$ & $697 \pm 51$ & $2.08 \times 10^{-5}$ \\
090328 & $(1.52 \pm 0.02) \times 10^{-4}$ & $\sim 25$ & $-0.93 \pm 0.02$ & $-2.2 \pm 0.1$ & $653 \pm 45$ & $1.415 \times 10^{-5}$ \\
$090510 \mathrm{~A}$ & $(2.3 \pm 0.2) \times 10^{-5}$ & 1 & $-0.80 \pm 0.03$ & $-2.6 \pm 0.3$ & $4400 \pm 400$ & $3.25 \times 10^{-7}\left(5.57 \times 10^{-7}\right)^{\mathrm{a}}$ \\
$090902 \mathrm{~B}$ & $(5.4 \pm 0.04) \times 10^{-4}$ & $\sim 21$ & $-0.696 \pm 0.012$ & $-3.85 \pm 0.25$ & $775 \pm 11$ & $6.05 \times 10^{-5}$ \\
$090926 \mathrm{~A}$ & $(1.9 \pm 0.05) \times 10^{-4}$ & $20 \pm 2$ & $-0.75 \pm 0.01$ & $-2.59 \pm 0.05$ & $314 \pm 4$ & $4.316 \times 10^{-5}$ \\
091003 & $(4.16 \pm 0.03) \times 10^{-5}$ & $21 \pm 0.5$ & $-1.13 \pm 0.01$ & $-2.64 \pm 0.24$ & $86.2 \pm 23.6$ & $2.279 \times 10^{-5}$ \\
\hline
\end{tabular}

Notes. The first six columns give the burst parameters as measured by the Fermi GBM (Ghisellini et al. 2010), including those for GRB 090510A, which was also localized by Swift BAT. The last column gives the predicted BAT fluences as extrapolated from the GBM parameters. The indices $\beta_{1 \mathrm{GBM}}$ and $\beta_{2 \mathrm{GBM}}$ are the low and high Band spectral parameters, respectively. Fluences, $S$, are given in ( $\mathrm{erg} \mathrm{cm}^{-2}$ ). We use a Band function for the GBM spectrum, with the exception of GRB 090323, for which a cutoff power-law model is adopted.

${ }^{a}$ Actual fluence observed by BAT.

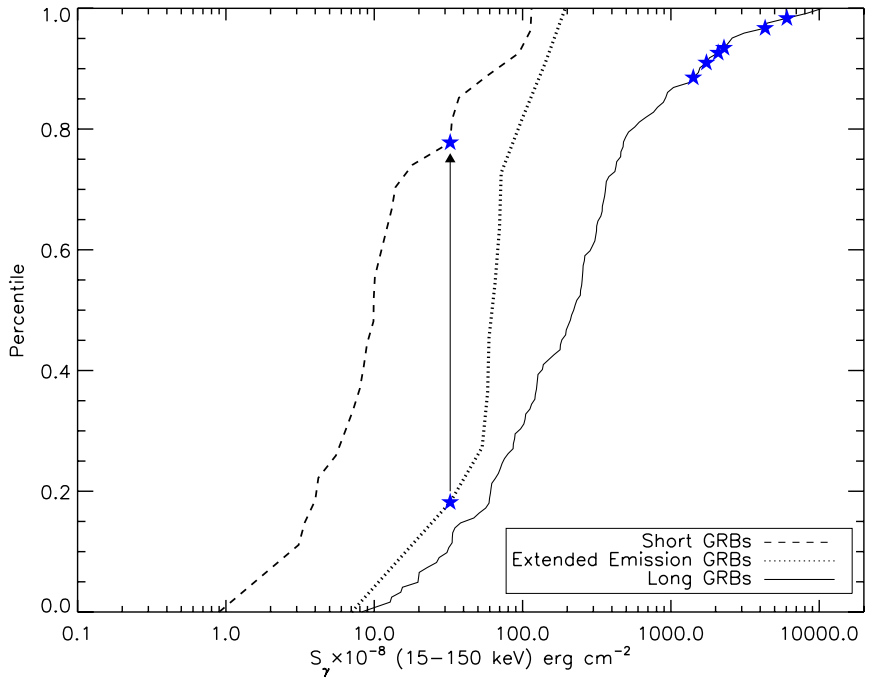

Figure 3. Distribution curve for 343 BAT bursts from 2005 April to 2009 June, and seven LAT bursts as a function of fluence. The stars indicate the LAT-detected GRBs, also observed by Swift, using the predicted BAT fluence. GRB 090510A is shown on both the short and extended emission curves, joined by an arrow.

(A color version of this figure is available in the online journal.)

fact present, GRB 090510A would be one of the lowest fluence extended emission bursts triggered by the BAT. If there was no extended emission associated with GRB 090510A, then it would be brighter than $\sim 77 \%$ of all BAT-triggered non-extended emission short bursts, making it a better corollary to the long LAT GRBs.

We have shown that long LAT-detected GRBs are brighter than $88 \%$ of BAT-triggered bursts and that the lone short burst is also brighter than $\sim 77 \%$ of other short bursts. To test whether this trend continues to the X-ray and UV/optical wavelengths, we also compared the afterglows of the LAT sample to BATtriggered bursts with XRT and UVOT afterglows. We compared the X-ray flux of LAT bursts at $\sim 70 \mathrm{ks}$ to a selection of 314 $\mathrm{X}$-ray light curves taken from the XRT light-curve repository (Evans et al. 2007, 2009). GRB 090510A was only detected by the XRT until $\sim 35 \mathrm{ks}$, so we used the flux at $35 \mathrm{ks}$ for comparing the short and extended emission bursts. We find the $\mathrm{X}$-ray afterglows of long LAT-triggered bursts are brighter than those of $80 \%$ of the BAT-triggered bursts, as shown in Figure 4. The X-ray afterglow of GRB 090510A is brighter than 64\% $(69 \%)$ of the BAT-extended emission (short) bursts.

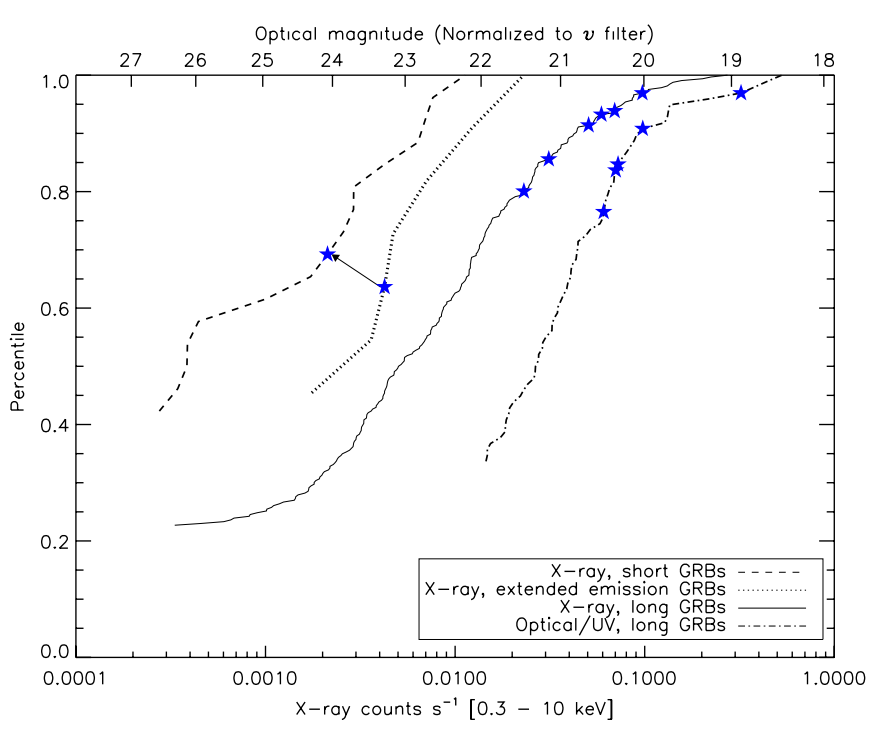

Figure 4. X-ray and optical distribution curves. X-ray curves using flux from 284 XRT afterglows. Long bursts flux taken at $70 \mathrm{ks}$, short and extended emission at $35 \mathrm{ks}$. Short burst curve is shifted to left by a factor of 2 , for clarity. GRB 090510A is shown on both the short and extended emission curves, joined by an arrow. Optical distribution curve in both counts $\mathrm{s}^{-1}$ and magnitudes in UVOT $v$ filter at $70 \mathrm{ks}$. Observations resulting in upper limits are not included. Stars indicate LAT bursts.

(A color version of this figure is available in the online journal.)

We compared the optical flux at $70 \mathrm{ks}$ to 103 bursts with UVOT afterglows included in The Second Swift UltraViolet/Optical Telescope GRB Afterglow Catalog (P. W. A. Roming et al. 2010, in preparation). All light curves were normalized to the $v$-filter and extrapolated to $70 \mathrm{ks}$ (if necessary) for our comparison. Our preliminary results, Figure 4, indicate that the optical afterglows of long LAT bursts are brighter than $77 \%$ of BAT-triggered optical afterglows, with GRB 090926A falling in the top $3 \%$ of optical afterglow brightness. Additionally, GRB 090510A is one of the only two extended emission GRBs, or one of the five short GRBs, still detected by the UVOT at $70 \mathrm{ks}$. Regardless of which category GRB 090510A belongs to, it is brighter than $\sim 90 \%$ of other short/extended emission optical afterglows.

\section{CONCLUSIONS}

We have presented the Swift and Fermi observations of GRB 090926A, a recent LAT-detected GRB with a bright, long-lived 
optical afterglow observed by UVOT. We have compared this burst to other LAT- and BAT-detected bursts in an attempt to show whether the GRBs detected by LAT are simply brighter than the average BAT-triggered GRB or whether they represent a new type of GRB that commonly exhibits bright, long-duration optical afterglows due to some form of energy injection.

We find that LAT-detected bursts are generally brighter than their BAT-triggered counterparts. We find that their fluence is consistently higher than the "average" BAT burst and that their $\mathrm{X}$-ray and UV/optical afterglows are brighter than $\sim 80 \%$ of BAT GRBs.

Although we are working with a small sample of LAT bursts, and therefore suffer from small number statistics, our preliminary results indicate that LAT bursts exhibit bright late-time X-ray and UV/optical afterglows because they are brighter at all wavelengths than the "average" burst, assuming the higher than average fluence can be extrapolated down to $\mathrm{X}$-ray and UV/optical wavelengths. This seems to be the most likely explanation, given the known correlation between prompt emission and afterglow emission brightness (Gehrels et al. 2008). We cannot say definitively, however, that this is the reason for the bright afterglows at late times, due to the presence of flares, which indicate possible late-time central engine activity that could cause a rebrightening. Without coverage of the early afterglow, it is impossible to say how the afterglow arrived at the state in which we observe it $\sim 70 \mathrm{ks}$ after the trigger. If we simply extrapolate the optical light curve of GRB 090926A backward, we find that they could have peaked as high as $v=10$ mag within the first hundred seconds after the trigger. Extrapolating the LAT spectrum of GRB 090926A to the $v$ band yields a peak magnitude of $v \approx 4$, or if we assume a cooling break at $\mathrm{GeV}$ energies, the spectral index changes to $\beta \approx-0.76$, yielding a magnitude of $v \approx 15$, consistent with our extrapolation backward and the idea that LAT bursts are uniquely bright at all wavelengths. However, if the early afterglow was fainter than $v \approx 15 \mathrm{mag}$, then some sort of sustained energy injection would be required to keep the flux elevated at a level where we could then observe the bright afterglow at $70 \mathrm{ks}$ after the trigger. Such an energy injection would test our current theoretical understanding of GRB optical afterglows. Our ability to determine the true nature of an LATdetected burst is contingent on our ability to follow-up LATdetected GRBs at earlier times than has been achieved with the current sample.

This work made use of data supplied by the UK Swift Science Data Centre at the University of Leicester.

\section{REFERENCES}

Amati, L., et al. 2002, A\&A, 390, 81

Atwood, W. B., et al. 2009, ApJ, 697, 1071

Band, D., et al. 1993, ApJ, 413, 281

Barthelmy, S. D., et al. 2005, Space Sci. Rev., 120, 143

Bissaldi, E. 2009, GCN, 9933, 1

Burrows, D. N., et al. 2005a, Space Sci. Rev., 120, 165

Burrows, D. N., et al. 2005b, Science, 309, 1833

Chincarini, G., et al. 2007, ApJ, 671, 1903

De Pasquale, M., et al. 2010, ApJ, 709, L146

Evans, P. A., et al. 2007, A\&A, 469, 379

Evans, P. A., et al. 2009, MNRAS, 397, 1177

Gehrels, N., et al. 2004, ApJ, 611, 1005

Gehrels, N., et al. 2008, ApJ, 689, 1161

Ghisellini, G., Ghirlanda, G., \& Nava, L. 2010, MNRAS, 403, 926

Gronwall, C., \& Vetere, L. 2009, GCN, 9938, 1

Kalberla, P. M. W., Burton, W. M., Hartmann, D., Arnal, E. M., Bajaja, E., Morras, R., \& Pöppel, W. G. L. 2005, A\&A, 440, 775

Krimm, H. A., et al. 2007, ApJ, 665, 554

Krimm, H. A., et al. 2009, ApJ, 704, 1405

Malesani, D., et al. 2009, GCN, 9942, 1

Margutti, R., et al. 2010, MNRAS, in press (arXiv:1004.1568v1)

Maxham, A., \& Zhang, B. 2009, ApJ, 707, 1623

McBreen, S., et al. 2010, A\&A, in press (arXiv:1003.3885v1)

Meegan, C., et al. 2009, ApJ, 702, 791

Oates, S. R., et al. 2009, MNRAS, 395, 490

Rau, A., et al. 2010, ApJ, submitted (arXiv:1004.3261v1)

Roming, P. W. A., et al. 2005, Space Sci. Rev., 120, 95

Roming, P. W. A., et al. 2009, ApJ, 690, 163

Uehara, T., Takahashi, H., \& McEnery, J. 2009, GCN, 9934, 1

Ukwatta, T. N., et al. 2009, GCN, 9337, 1

Wu, X. F., Dai, Z. G., Wang, X. Y., Huang, Y. F., Feng, L. L., \& Lu, T. 2005, arXiv:astro-ph/0512555v1

Yonetoku, D., et al. 2004, ApJ, 609, 935

Zhang, B. 2007, Chin. J. Astron. Astrophys., 7, 1

Zhang, B., \& Mészáros, P. 2002, ApJ, 581, 1236

Zhang, B., et al. 2006, ApJ, 624, 354 